\title{
High Time-Resolution Astronomy on the 10-m SALT
}

\author{
Barry Welsh $^{1}$, David Anderson ${ }^{1}$, Jason McPhate ${ }^{1}$, John Vallerga ${ }^{1}$, \\ Oswald H. W. Siegmund ${ }^{1}$, David Buckley ${ }^{2}$, Amanda Gulbis ${ }^{2}$, \\ Marissa Kotze ${ }^{2}$ and Stephen Potter ${ }^{2}$ \\ ${ }^{1}$ Experimental Astrophysics Group, Space Sciences Laboratory, UC Berkeley, CA 94720, USA \\ email: bwelsh@ssl. berkeley.edu \\ ${ }^{2}$ SALT, South African Astronomical Observatory, Cape Town, South Africa
}

\begin{abstract}
We present a brief description of the Berkeley Visible Imaging Tube (BVIT) detector system, which is a user instrument on the 10-m Southern African Large Telescope (SALT), and include some preliminary observational results gained in mid-2011. The data show that BVIT is capable of revealing emission features occurring on time-scales of $<0.1 \mathrm{sec}$, thus opening up for the general user a window of high time-resolution astronomy at visible wavelengths.
\end{abstract}

Keywords. detector, transients

\section{Introduction}

The recording of emission from astronomical objects on time-scales of $<1$ sec has generally not been possible at frequencies other than gamma-ray, X-ray or radio. (However, we note the exception of the ULTRACAM instrument: Dhillon \& Marsh 2001). Although the next decade will be devoted to many surveys of the sky recorded (like PSST or PanSTARRS) on time-scales of days to weeks, sparse attention has been focused on the visible variability of objects over very short time-periods. However, the Berkeley Visible Image Tube (BVIT) fills this void by allowing users to perform differential photon-counting photometric observations of faint objects at visible wavelengths with time-resolutions of micro-seconds. The BVIT (Siegmund et al. 2008) is currently a user-instrument on the 10-m Southern African Large Telescope (SALT, Buckley et al. 2010), and is ideally suited for making observations of rapid astrophysical emission phenomena associated with flare stars, low-mass X-ray binary systems, optical pulsars and cataclysmic variables. In addition, because of the high cadence of observations, BVIT can also be used in the accurate timing of eclipses in AM Her systems, exo-planet transits and stellar occultations by small solar-system bodies such as Kuiper Belt Objects. Details about both the instrument and its observational performance can be found at http://bvit.ssl.berkeley.edu.

\section{The BVIT Instrument}

BVIT is a simple instrument with minimal observational setup requirements and a high degree of post-acquisition data flexibility. At its core is a 25 -mm-diameter microchannel plate sealed tube detector, whose SuperGen-2 photocathode converts incident visible photons into photoelectrons that are amplified by gains of up to $10^{8}$. The resultant charge cloud is accelerated onto a delay-line anode which enables the position $(\mathrm{x}, \mathrm{y})$ and time of arrival (t) to be recorded. Similar detection schemes are currently being flown on the NASA GALEX and HST-COS missions. In Fig. 1 we show the entire BVIT instrument in its self-contained enclosure on the SALT; it includes dual $B, V, R, \mathrm{H} \alpha$ 


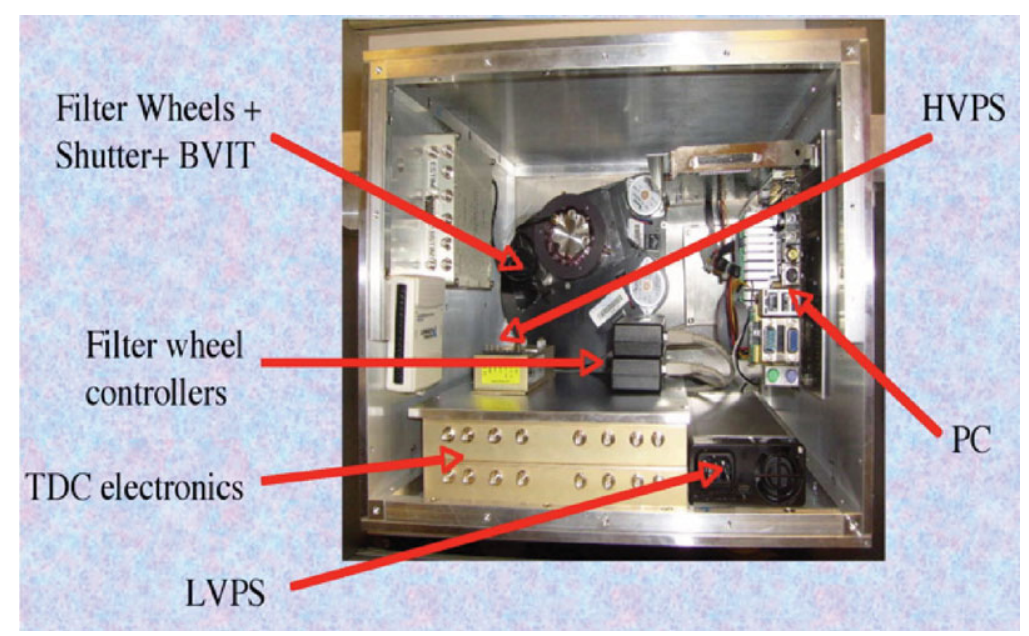

Figure 1. The BVIT detector system on SALT

and ND filter wheels, an adjustable field iris (1'.9 field of view), a field shutter and the support electronics for the detector system (HVPS, LVPS, Xilinx based time-to-digital board and a mini-ITX PC with 0.5 Tera-byte hard disk).

The 1'.9 field of view allows real-time monitoring of both the target and the skybackground time-tagged fluxes, thus enabling discrimination between fast transient events seen in the target and/or background signals. The ability to perform time-tagged photon flux monitoring of comparison stars lying within the detector field of view enables relative (differential) flux photometric comparison throughout the entire observation. Typically, sources of visible magnitude in the range $V=12.5$ to 22.0 can be observed with high time-resolution on the SALT with BVIT.

\section{BVIT Observations}

BVIT observations on the SALT are currently funded by a 3-year NSF research grant. The main scientific objectives are simultaneous monitoring of low-mass X-ray binary systems with the RXTE satellite, AM Her-type eclipse monitoring, exo-planet transit timing and the search for optical counterparts to the Rotating Radio Transients (RRATs) (McLaughlin et al. 2006).

In this section we report on data recorded over a 4-night period (2011 June 2-6) on the SALT. Time-tagged photons were extracted in a selected radius around the position of the target, in addition to similarly selected time-tagged photons from a comparison star within the detector field of view and also photons from the sky background. The target data were then corrected for variations in the comparison star and sky background signals as a function of both time and position, to account for variable seeing/focus and any image tracking drifts. In Fig. 2 we show the results of that analysis process for the AM Her system of UZ For, displaying the egress from an eclipse at $\mathrm{t} \sim 400$ sec.

\subsection{GR Mus}

GR Mus (4U 1254-690) is a well-observed low-mass X-ray binary system (Barnes et al. 2007) that presently is in its "low-phase" of emission activity. In their "high" states these systems often show fast flickering on time-scales as short as $20 \mathrm{~ms}(50 \mathrm{~Hz})$, together with longer-term eruptive events and quasi-periodic oscillation (QPO) events with frequencies less than $1 \mathrm{~Hz}$. Those processes are linked to the transfer of mass from the companion 


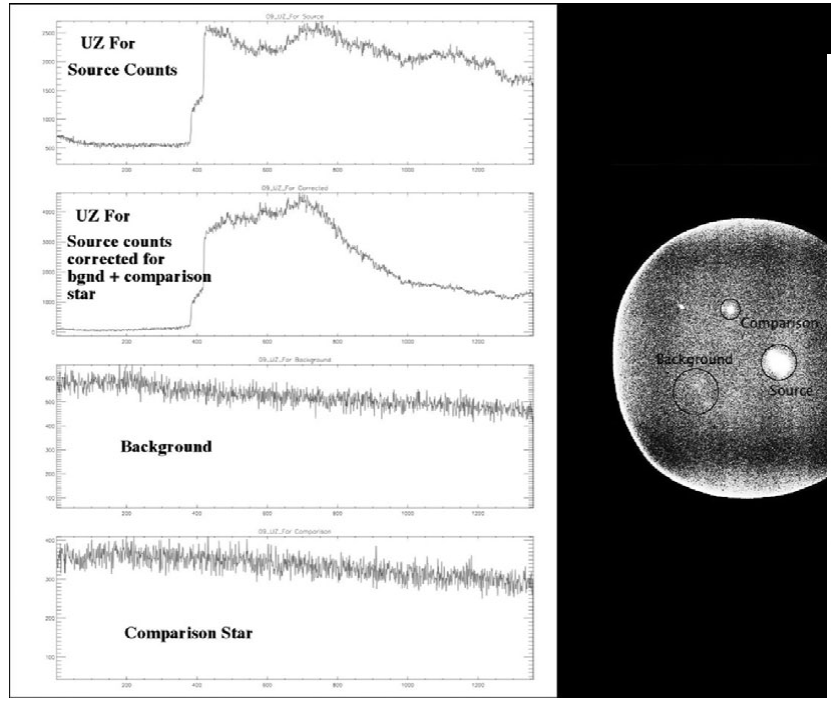

Figure 2. Right: BVIT image of the UZ For sky field. Left: Plots of flux versus time for the target star (raw and corrected), comparison star and sky background.

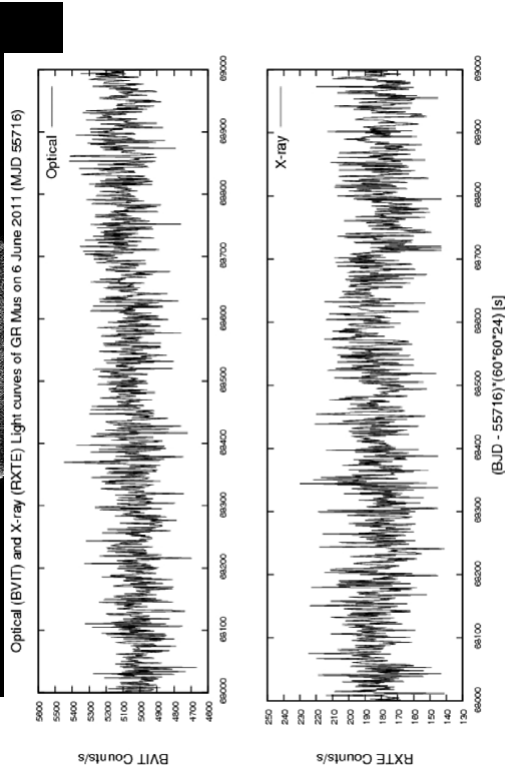

Figure 3. Plots of BVIT visible and RXTE X-ray flux versus time for GR Mus.

star to the accretion disk surrounding the central black hole (or neutron star). It has been shown by Shih et al. (2011) that the associated optical emission varies on the same timescale as, and is correlated with, the soft X-ray emission, and demonstrates that X-ray reprocessing in such systems is mainly driven by soft X-rays. The presence of many QPOs and breaks in the frequency power-density spectrum of such X-ray binaries is thought to indicate the characteristic time-scale at some transition radius in the accretion disk.

In Fig. 3 we show flux versus time plots for thousands of data points recorded simultaneously with BVIT and the RXTE satellite on 2011 June 6. Both data sets confirm the very low state of activity of this source at present. We find no evidence for QPO activity in the power density spectrum of the BVIT data, and a cross-correlation analysis results in a null result (correlation $< \pm 0.1$ ) in terms of time-lags between emission in the visible and X-ray data. A full description of the treatment of these data is being prepared for publication.

\subsection{CN Leo}

CN Leo is a very active M6 flare star known for its recurrent flaring activity observed at radio, visible, UV and X-ray wavelengths (Fuhrmeister et al. 2008). In Fig. 4 we show B-band BVIT observations of a rarely seen double flare event occurring within a 500-sec time period on CN Leo. This double-flare takes $\sim 1500$ sec decay time to return to the flux level recorded immediately prior to the first flare. White-light flare emission is thought to consist primarily of blackbody radiation at the flare peak and hydrogen continuum during the decay time period (Zhilyaev et al. 2007), and as such, our B-band data are unable unambiguously to differentiate between those spectral components as a function of the flare's evolution with time. However, the high time-resolution capability of BVIT is able to reveal at least two prominent cases of pre-flaring at $t=63 \mathrm{sec}$ and $74 \mathrm{sec}$ ( $\mathrm{see}$ Fig 5). In addition, radiative hydrodynamic models of short-lived ( $\sim 15 \mathrm{sec})$ M-dwarf flares suggest that variations in the emitted flux should appear over time-frames as short 


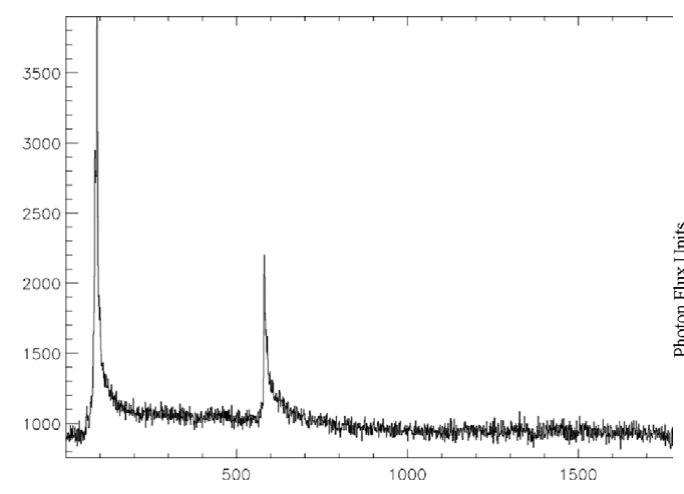

Figure 4. Double flare event seen on CN Leo with B-band BVIT observations; data binned at 1 sec.

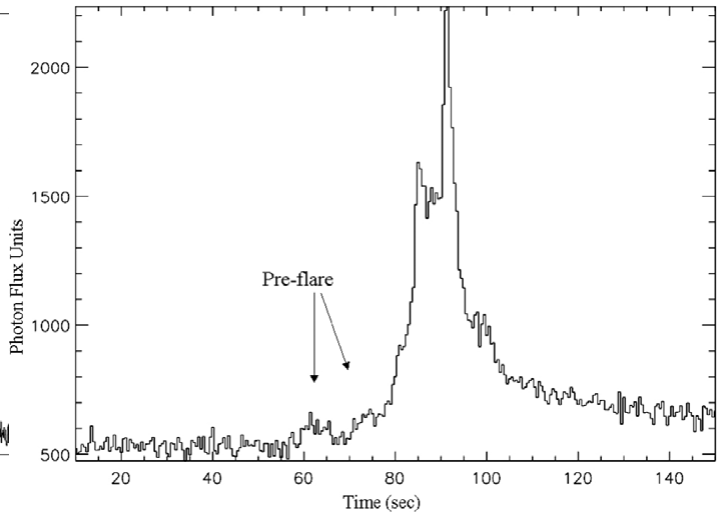

Figure 5. Pre-flare activity on CN Leo revealed by BVIT.

as $0.1 \mathrm{sec}$ (Allred et al. 2006), whereas previous visible observations suggested that no fine structure in emitted flux was evident on time-scales less than $0.3 \mathrm{sec}$ (Beskin et al. 1990). The new BVIT data, when binned at 0.01-sec intervals, show emission structure at or below the 0.1-sec level. The BVIT data also reveals a small dip in the emitted flux at 1370 sec. Such diminutions in the stellar continuum after a flare event have been previously reported (Hawley et al. 1995) and may be due to the initial flare energy impacting on the photosphere, which leads to an increase in the hydrogen opacity and thus causing a small drop in the observed emitted flux.

\section{Conclusion}

Although BVIT has only been initially tested on the SALT, these early data and performance characteristics are encouraging in that it is clear that complex emission processes have signatures that can only be revealed through high time-resolved observations with cadences $<0.1$ seconds. In addition, such high cadences are also important for the precise timing of various eclipse phenomena, especially exo-planet transits where variations in the eclipse ephemeris predictions could indicate the presence of other, unseen planetary objects.

\section{References}

Allred, J., et al. 2006, ApJ, 644, 484

Barnes, A., et al. 2007, MNRAS, 380, 1182

Beskin, G., et al. 1990, BCrAO 79, 67

Buckley, D., et al. 2010, Proc. SPIE, 7735, 174

Dhillon, V. \& Marsh, T. 2001, New. Astron. Revs, 45, 91

Fuhrmeister, B., Liefke, C., Schmitt, J., \& Reiners, A. 2008, A\&\&A, 487, 293

Hawley, S., et al. 1995, ApJ, 453, 464

Kotze, M., et al. 2012 MNRAS, in preparation

McLaughlin, M., et al. 2006, Nature, 439, 817

Shih, I., Charles, P., \& Cornelisse, R. 2011, MNRAS, 412, 120

Siegmund, O. H. W., et al. 2008, in: D. Phelan, O. Ryan \& A. Shearer (eds.), High Time Resolution Astrophysics. AIP Conf. Ser. Vol. 984, (AIP), p. 103

Zhilyaev, B., et al. 2007, A\&A, 465, 235 